\title{
ANÁLISE DE CLASSIFICADORES DE ESTÁGIOS SUCESSIONAIS EM UM FRAGMENTO DE MATA ATLÂNTICA
}

\author{
ANALYSIS OF SUCCESSIONAL CLASSIFIERS IN A FRAGMENT OF ATLANTIC FOREST
}

\author{
Gabriela Miranda Teixeira ${ }^{1}$, Pablo Hugo Alves Figueiredo², Silvio Frosini de Barros Ferraz ${ }^{3}$, Luiz \\ Felippe Salemi ${ }^{4}$, Maurício Ranzini ${ }^{5}$, Nivaldo Eduardo Rizzi ${ }^{6}$ \\ 1,6Universidade Federal do Paraná, Curitiba, Brasil-gabrielateixeirabio@gmail.com \& niva@ufpr.br \\ ${ }^{2}$ Escola Nacional de Botânica Tropical, Rio de Janeiro, Brasil-pablo_figueiredo@yahoo.com.br
}

${ }^{3}$ Escola Superior de Agricultura "Luiz de Queiroz", Piracicaba, São Paulo, Brasil - silvio.ferraz@usp.br

${ }^{4}$ Universidade de Brasilia, Distrito Federal, Brasil - Ifsalemi@unb.br

5Insituto Florestal do Estado de São Paulo, São Paulo, Brasil - ranzini@gmail.com

\section{RESUMO}

Apesar da reconhecida importância da Mata Atlântica, há uma escassez de estudos utilizando o sensoriamento remoto como ferramenta para identificação e classificação dos diferentes estágios sucessionais de seus remanescentes florestais. Neste estudo comparamos o desempenho de diferentes métodos para classificação de estágio sucessional e investigamos a existência de sazonalidade na resposta espectral de uma floresta tropical densa na Mata Atlântica. Usamos amostras de treinamento de três estágios sucessionais obtidas a partir de uma ortofoto de 2010 e selecionamos imagens Landsat 5 TM para os anos de 2009, 2010 e 2011, considerando os meses de maiores e menores médias históricas de temperatura e precipitação. Para avaliação da sazonalidade da resposta espectral usamos o teste de Mann-Whitney, comparando cada banda do espectro eletromagnético e estágios sucessionais entre as épocas de aquisição das imagens. Para classificação da cobertura vegetal usamos três índices de vegetação (NDVI, EVI e NDMI) e Análise Discriminante Quadrática (QDA). Comparamos a acurácia dos classificadores a partir de matrizes de validação cruzada. Nossos resultados mostram diferenças significativas entre os estágios sucessionais para todas as bandas espectrais, com melhor distinção na época de menores temperaturas e precipitação. QDA foi o classificador com maior acerto global (92\%), seguido por NDMI (68\%), NDVI (67\%) e EVI (59\%). Concluímos que QDA é, dentre os classificadores avaliados, o mais eficiente para classificação sucessional da floresta e que imagens obtidas em época de menor precipitação e temperatura geram uma melhor distinção entre estágios sucessionais para essa fisionomia florestal.

PALAVRAS-CHAVE: Análise discriminante quadrática, Floresta tropical, Índices de vegetação.

\section{ABSTRACT}

Despite the recognized importance of the Atlantic Forest, there is a shortage of studies using remote sensing as a tool to identify and classify the different successional stages of its forest remnants. In this study we compared the performance of different classifiers on the determination of successional stages and investigated the existence of seasonality in the spectral response of a dense tropical Atlantic Forest area. We used training samples of three successional stages gathered by an orthophoto from the year of 2010 and selected Landsat 5 TM images for the years of 2009, 2010 and 2011, considering the months of higher and lower historical averages of temperature and precipitation. To evaluate the seasonality in forest spectral response we used the Mann-Whitney test, comparing each band and successional stage between the two studied periods. To classify the vegetation cover we used three indices (NDVI, EVI and NDMI) and a Quadratic Discriminant Analysis (QDA). We compared classifiers accuracy using a cross validation matrix. We found significative differences between successional stages on all electromagnetic spectral zones, with finer definitions between stages at the samples of colder and drier periods. QDA was the classifier with higher global accuracy (92\%), followed by NDMI (68\%), NDVI (67\%) and EVI (59\%). We concluded that QDA is the most efficient classifier among the ones studied for forest succession and that satellite images taken during colder and drier season show better distinction among successional stages for this forest physiognomy. 


\section{INTRODUÇÃO}

Considerada um dos hotspots mundiais de biodiversidade (GARDNER et al., 2010), a Mata Atlântica é um dos biomas brasileiros que mais sofreu com as atividades antrópicas, estando hoje constituída por fragmentos florestais predominantemente pequenos e em diferentes estágios de sucessão (RIBEIRO et al., 2009). Por esse motivo, o conhecimento do desenvolvimento e da dinâmica temporal da cobertura vegetal é uma valiosa ferramenta para o planejamento da paisagem e para decisões sobre estratégias de conservação e restauração dos recursos florestais.

Atualmente, existem diversos estudos dedicados à medição das características biofísicas dos fragmentos florestais visando documentar a biodiversidade e a integridade estrutural e funcional dos mesmos. Informações desta ordem são imprescindíveis e fundamentais, entretanto, essas medições geralmente dependem de um trabalho de campo extenso e oneroso, além de abranger áreas de estudo restritas. Neste contexto, as técnicas de sensoriamento remoto apresentam grande potencial para o mapeamento e avaliação das condições estruturais dos recursos florestais, permitindo monitorar áreas amplas e em períodos de tempo constantes (DEVRIES et al., 2015).

Apesar da reconhecida importância da Mata Atlântica, há uma escassez de estudos utilizando o sensoriamento remoto como ferramenta para identificação e classificação dos diferentes estágios sucessionais de seus remanescentes e suas florestas secundárias. A maior parte dos estudos disponíveis indicam apenas a existência, ou não, de cobertura florestal, sem fornecer indicações de sua estrutura e maturidade, o que, portanto, restringe sua utilização em pesquisas de dinâmica florestal, ecologia e planejamento de conservação.

Os Índices de Vegetação (IV) obtidos de sensoriamento remoto têm sido extensivamente usados em estudos de detecção de mudanças na cobertura florestal (ALVES et al., 2009; IZQUIERDO et al., 2011; DEVRIES et al., 2015; SCHULTZ et al., 2016). Nas florestas tropicais, os IV foram associados à diversidade de espécies arbóreas, à biomassa e maturidade florestal.

Atualmente, existe uma grande diversidade de IV, os quais são obtidos a partir da recombinação matemática de valores de respostas espectrais dos alvos na superfície. Imagens da série Landsat, devido à boa resolução espacial e à alta disponibilidade de dados históricos, têm sido amplamente utilizadas para obtenção de IV e monitoramento da cobertura florestal (ALVES et al., 2009).
A resposta espectral da cobertura vegetal é influenciada por condições climáticas, ambientais e ecofisiológicas, podendo variar sazonalmente de acordo com tais fatores. Desse modo, os valores espectrais também podem ser empregados na avaliação dos aspectos sazonais de formações florestais (ZHANG et al., 2017).

Considerando que, de uma maneira geral, os IV utilizam as informações de duas ou três bandas do espectro eletromagnético e que os alvos na superfície terrestre interagem com todas as bandas disponíveis, acredita-se que análises que considerem um maior número de bandas para identificação e classificação da cobertura florestal apresentem melhores eficiências.

Desta forma, os objetivos deste estudo foram: (i) distinguir estágios sucessionais em Floresta Ombrófila Densa por meio de classificação de imagens Landsat 5 TM; (ii) verificar a presença de sazonalidade da resposta espectral em área onde não ocorre déficit hídrico ao longo do ano; e (iii) comparar diferentes métodos de classificação de imagens para identificação de estágios sucessionais da floresta.

\section{MATERIAL E MÉTODOS}

\section{Área de estudo}

O estudo foi desenvolvido no Parque Estadual da Serra do Mar (PESM), núcleo Cunha, localizado entre as coordenadas $45^{\circ} 02^{\prime} \mathrm{W}$ e $23^{\circ} 25^{\prime} \mathrm{S}$ (Figura 1 ).

De acordo com a classificação climática de Köppen, o clima predominante na região é do tipo Cwb, ou seja, temperado chuvoso e moderadamente quente (RANZINI et al., 2011), com temperatura média anual de $17^{\circ} \mathrm{C}$ (ARMANI, 2004). A proximidade da Serra do Mar com o Oceano Atlântico permite a ocorrência de pluviosidade mesmo no inverno, com precipitação média anual de 2.000 mm (ARCOVA et al., 2016).

Distante $15 \mathrm{~km}$ do litoral norte do Estado de São Paulo e voltado para vertente continental da Serra do Mar (ARMANI, 2004), o núcleo Cunha situa-se em uma região montanhosa, com declividades das encostas superiores à $15^{\circ}$, onde configura-se a aparência de "mares de morros" (FURIAN \& PFEIFER, 1986). As classes de níveis de altitude são distribuídas, num plano geral, entre 1.000 e $1.228 \mathrm{~m}$ (FURIAN, 1987).

A formação geológica da área pertence ao domínio tectônico do Terreno Embu, formada por depósitos terrígenos de origem incerta do Neoproterozóico ou PréCambriano, com predomínio de gnaisses e presença esparsa de migmatitos e granitos (FURIAN \& PFEIFER, 
1986). Essas rochas duras, de difícil decomposição, deram origem aos solos, classificados por Furian \& Pfeifer (1986), como Latossolo Vermelho Amarelo Câmbico. Estes são ácidos e pobres em minerais, apresentam textura grosseira, elevada porosidade e boa permeabilidade, sendo susceptíveis a erosão e a escorregamentos (CICCO, 2009).

O núcleo Cunha está inserido nos domínios do bioma Mata Atlântica e constitui uma área de Floresta Ombrófila Densa Montana (AGUIAR et al., 2001). Segundo o Manual Técnico da Vegetação Brasileira (IBGE, 2012), as formações montanas correspondem, na Região Sul do País, à vegetação situada entre 400 e $1.000 \mathrm{~m}$ de altitude. Apesar do núcleo estar situado em altitude superior a $1.000 \mathrm{~m}$ (FURIAN, 1987), a fitofisionomia local se assemelha mais à formação Montana, uma vez que nessa faixa latitudinal as características climáticas necessárias à conformação de ambientes Alto-Montanos ocorrem em altitudes mais elevadas.

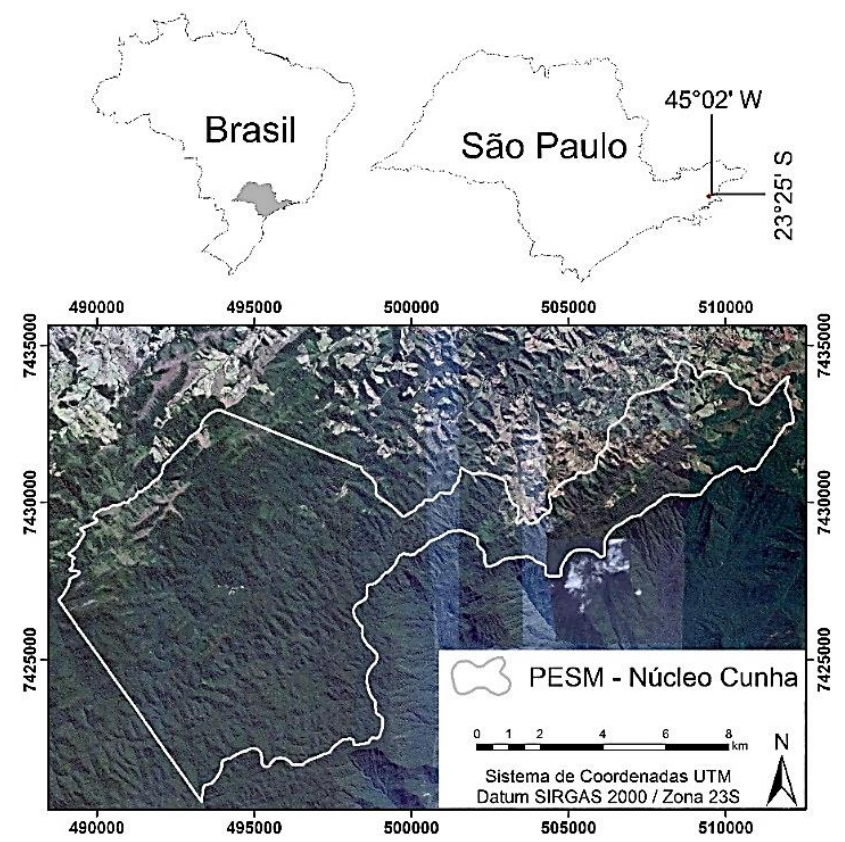

Figura 1. Localização do Núcleo Cunha do Parque Estadual da Serra do Mar (PESM - Cunha), São Paulo, Brasil.

Em caracterização da vegetação em uma microbacia localizada no núcleo Cunha, Aguiar et al. (2001) observaram a presença de três estratos verticais bem definidos: um sub-bosque relativamente fechado, um estrato intermediário com árvores de 10 a $14 \mathrm{~m}$ e um dossel com árvores de 16 a $20 \mathrm{~m}$ com ocorrência de alguns indivíduos emergentes com altura em torno dos $23 \mathrm{~m}$. Estes dados concordam com a caracterização de ambientes Montanos (IBGE, 2012).
A área, anteriormente denominada Fazenda São Lourenço, foi desapropriada em 1974 pelo Governo do Estado de São Paulo para criação da Reserva Estadual de Cunha que, em 1977, foi incorporada ao Parque Estadual da Serra do Mar (CICCO, 2009). Antes da consolidação da área como Unidade de Conservação, a vegetação local foi submetida à exploração para produção de carvão e madeira para serraria, sendo parcialmente substituída por pastagens e agricultura de subsistência (AGUIAR et al., 2001). Em locais de difícil acesso, como encostas declivosas e topos de morro a vegetação foi preservada, bem como em algumas regiões de fundo de vale (CICCO, 2013). Nas áreas exploradas, hoje predomina uma vegetação secundária em franco processo de regeneração natural (RANZINI et al., 2011).

\section{Base de dados}

Como verdade terrestre utilizou-se uma ortofoto digital colorida do ano de 2010 com resolução espacial de $1 \mathrm{~m}$, a qual foi elaborada pela Base Aerofoto e cedida pela equipe de geoprocessamento do Instituto Florestal de São Paulo.

Para classificação da vegetação e desenvolvimento das análises foram utilizadas imagens do satélite Landsat 5 TM, cena 218/76, para os anos de 2009, 2010 e 2011, as quais foram adquiridas junto ao United States Geological Survey (USGS). A utilização de imagens de um ano anterior e um posterior à ortofoto de referência se deu com o intuito de aumentar o tamanho das amostras de treinamento e por considerar que durante esse período as alterações na vegetação foram pequenas, visto que se trata de uma unidade de conservação e sua respectiva zona de amortecimento.

A seleção das imagens considerou o mês de obtenção e a qualidade das mesmas. Foram selecionadas imagens de duas diferentes épocas do ano. Ao todo foram obtidas três imagens para o período com maiores médias históricas de temperatura e precipitação (datas de obtenção: $19 / 02 / 2009$, 06/02/2010 e 22/02/2010) e seis imagens para o período de menor intensidade de chuvas e menores temperaturas (datas de obtenção: 13/07/2009, 30/08/2009, 14/06/2010, 01/08/2010, 17/06/2011 e 04/08/2011).

\section{Pré-processamento das imagens}

Inicialmente as imagens do satélite Landsat 5 TM foram reprojetadas para Datum WGS 1984 UTM Zone 23S, visto que as imagens disponibilizadas pelo USGS, apesar de já georreferenciadas, são orientadas ao norte verdadeiro. 
Para atenuar a interferência atmosférica nas imagens foi realizada uma correção a partir do método DOS (dark object subtraction) melhorado (CHAVEZ, 1988). Na sequência, as imagens passaram pelo processo de calibração radiométrica, o qual transforma os números digitais (ND) corrigidos em valores de reflectância da superfície. Para conversão dos valores de ND em radiância espectral utilizou-se a metodologia proposta por Markham \& Barker (1987) e para o cálculo da reflectância a partir da radiância espectral utilizou-se a metodologia de Chander et al. (2009). Todas as etapas de pré-processamento das imagens foram realizadas no ERDAS Imagine 2014.

\section{Coleta de amostras de treinamento}

Devido à dificuldade de uma classificação mais precisa quanto aos inúmeros estágios de desenvolvimento da vegetação, optou-se por considerar apenas três classes, sendo elas: inicial, médio e avançado. Considerou-se como estágio inicial as áreas de campos antrópicos, com indivíduos arbóreos ausentes ou isolados, sem a formação de um dossel florestal e com predomínio de vegetação arbustiva e herbácea. $O$ estágio médio consistiu de áreas com predominância de espécies arbóreas formando um dossel definido, porém com baixa estratificação, subbosque denso e altura inferior às áreas definidas como avançadas. Este último estágio caracterizou-se por áreas com elevada riqueza e abundância de epífitas, elevada estratificação, com ocorrência de plantas emergentes e sub-bosque menos expressivo que no estágio médio.

A caracterização dos estágios foi realizada em campanha de campo realizada no ano de 2017. Foram obtidos 50 pontos para cada estágio sucessional com o auxílio de um GPS modelo Garmin MAP76 CSX. Esses pontos foram posteriormente inseridos sobre uma imagem de satélite de alta resolução obtida também em 2017 (Google Earth). Essa etapa teve como objetivo reconhecer as características visuais de cada estágio sucessional em imagem de alta resolução, de modo a auxiliar na interpretação da ortofoto obtida no ano de 2010.

Desse modo, como amostras de treinamento foram distribuídos 300 pontos sobre a ortofoto do ano de 2010, sendo 100 para cada um dos três estágios sucessionais considerados. Os pontos foram alocados no interior do núcleo Cunha e também em áreas adjacentes, respeitando-se um raio de $4 \mathrm{~km}$ da sede do núcleo.

As amostras de treinamento foram então sobrepostas às imagens Landsat 5 TM já corrigidas de forma a obter tabelas com valores de reflectância de cada banda para os pontos de estágio sucessional conhecido. Apenas as informações referentes à banda 6 não foram utilizadas por se tratar de uma banda termal.

\section{Comparação das épocas de aquisição das imagens}

Para avaliação da sazonalidade na resposta espectral da cobertura vegetal foram realizadas comparações entre as épocas de aquisição das imagens para cada banda e classe sucessional. Devido a não normalidade dos dados, todas as comparações foram realizadas com o teste Mann-Whitney, disponível no ambiente computacional $\mathrm{R}$ - função wilcox.test $(x, y$, paired $=F A L S E)$, com intervalo de confiança de $99 \%$.

\section{Comparação dos classificadores da cobertura vegetal}

Para classificação da cobertura vegetal foram utilizados três IV (Normalized Difference Vegetation Index - NDVI, Enhanced Vegetation Index - EVI e Normalized Difference Moisture Index - NDMI) e Análise Discriminante Quadrática (QDA). Tais técnicas foram aplicadas sobre as três imagens de melhor qualidade obtidas para o período com menor intensidade de chuvas e menores temperaturas (30/08/2009, 01/08/2010 e 04/08/2011). A escolha destas imagens se deu pela melhor distinção entre as classes sucessionais observadas neste período. Dessa forma, totalizou-se 300 amostras por estágio sucessional para cada técnica de classificação utilizada.

Devido a não normalidade dos dados, as comparações dos IV obtidos para cada classe sucessional foram realizadas com o teste Mann-Whitney, disponível no ambiente computacional $\mathrm{R}$ - função wilcox.test $(x, y$, paired $=F A L S E$ ), com intervalo de confiança de $99 \%$.

Para classificação da cobertura vegetal nos três estágios considerados neste estudo, obteve-se o valor médio dos três IV para cada classe sucessional e realizouse uma pós-classificação das amostras de treinamento, sendo atribuída ao pixel a classe com valor médio mais próximo a ele. Após a classificação foi gerada uma matriz de validação cruzada, a partir da qual verificou-se a acurácia de cada IV como classificador da vegetação.

Os dados de reflectância das seis bandas analisadas (1 a 5 e 7) também foram submetidos a uma Análise Discriminante Quadrádica (QDA). Utilizou-se a QDA pois os dados não atendiam ao pressuposto de homogeneidade de matrizes de covariância necessário à Análise Discriminante Linear. Esse pressuposto foi verificado através da função betadisper disponível no pacote Vegan do ambiente $\mathrm{R}$, um teste multivariado análogo ao teste de Levene para homogeneidade de variâncias. 
No caso do classificador quadrático, estima-se para cada classe um vetor de média e uma matriz de covariância próprios, sendo, portanto, indicado quando as matrizes covariância de cada classe são suficientemente distintas (MANLY, 2008). Como resultado são obtidas funções de decisão que separam as regiões distintas no espaço multiespectral correspondente a cada classe. Suas fronteiras de decisão assumem a forma de superfícies (ou hiper-superfícies) quadráticas, como elipsóides (ou hiperelipsóides), justificando o nome atribuído à técnica (THESSLER et al., 2008).

A QDA, como outras técnicas de análise multivariada, permite a discriminação entre categorias pelo estabelecimento de pesos às variáveis estatísticas, visando maximizar a variância entre os grupos e minimizando a variabilidade intraclasses (IMANI \& GHASSEMIAN, 2015). A possibilidade de que a resposta espectral das bandas auxilie na discriminação dos estágios sucessionais baseiase no pressuposto de que cada classe apresenta características de reflectância que a difere das demais e, portanto, seria possível utilizar estas variáveis para separação dos estágios.

A acurácia do classificador foi verificada a partir da porcentagem de alocações corretas observadas na matriz de validação cruzada, a qual foi construída a partir do método de classificação Jacknife de indivíduos. Este método aloca cada indivíduo ao seu grupo mais próximo sem usar aqueles indivíduos para ajudar a determinar o centro do grupo (MANLY, 2008).

\section{RESULTADOS E DISCUSSÃO}

\section{Sazonalidade da resposta espectral}

Para as três classes sucessionais houve predominância de bandas com valores de reflectância significativamente diferentes entre as épocas avaliadas ( $p$-valor $<0,01$; Tabela 1). Para o estágio avançado apenas a banda 1 (azul) não diferiu entre as estações. Para o estágio médio não foi verificada variação sazonal para as bandas 3 (vermelho) e 5 (infravermelho médio). Já para o estágio inicial não houve diferença para a banda 2 (verde). As bandas 4 (infravermelho próximo) e 7 (infravermelho médio) foram sazonalmente diferentes para todos os estágios sucessionais.

Para as duas épocas avaliadas foram encontradas diferenças significativas ( $p$-valor $<0,01$ ) entre as classes sucessionais para todas as bandas do espectro eletromagnético (Tabela 1). No entanto, de acordo com a Figura 2 podemos observar que as diferenças entre as classes sucessionais são mais acentuadas para o período de junho a agosto, quando são observadas menores temperaturas e taxas de precipitação. Para este período, com exceção da banda 4, 50 \% das observações de cada estágio apresentam boa separabilidade, o que pode ser percebido pela ausência de sobreposição em suas distribuições.

Apesar de se tratar de um ambiente onde não ocorre um período de déficit hídrico ao longo do ano, a pequena sazonalidade climática existente é suficiente para gerar diferenças na resposta espectral da cobertura vegetal. É bem conhecido que o teor de água das folhas é determinante na reflectância do infravermelho médio (LIN et al., 2015), o que explica, em parte, as diferenças encontradas para imagens adquiridas em períodos com diferentes quantidades de chuva. Este fato reforça a importância de se trabalhar com imagens adquiridas em uma mesma época do ano para estudos de dinâmica da cobertura vegetal, uma vez que a disponibilidade hídrica do solo poderá interferir na reflectância da vegetação na região do infravermelho.

A melhor separabilidade dos estágios sucessionais durante os meses de junho a agosto pode ser explicada pela diferença das respostas da vegetação às restrições climáticas impostas nessa época do ano. Durante os meses de dezembro a fevereiro, além da maior disponibilidade hídrica, ocorrem maiores taxas de insolação e menor ocorrência de nevoeiros (ARCOVA et al., 2016), o que permite que todas as classes sucessionais estejam fotossinteticamente ativas. Em contrapartida, as menores taxas de precipitação observadas de junho a agosto são sentidas de maneiras diferentes conforme a maturidade da vegetação. De acordo com Nepstad et al. (1994), florestas com sistema radicular profundo são menos sensíveis à ausência de chuvas, pois conseguem manter as taxas de fotossíntese e transpiração através da absorção de água presente em altas profundidades do solo.

\section{Índices de vegetação}

De acordo com a Tabela 2, verifica-se que o NDVI foi o índice que gerou os maiores valores para os três estágios, apresentando uma boa separação entre o estágio inicial e as demais classes. Porém, não foi eficiente para distinção entre os estágios médio e avançado. A distinção entre os estágios médio e avançando obtida pelo EVI foi superior à do NDVI. No entanto, este índice não foi eficiente para distinção entre os estágios avançado e inicial. O NDMI foi o único índice em que todos os estágios diferiram significativamente entre si. 
Tabela 1. Média e desvio padrão da resposta espectral de cada banda, para cada classe sucessional e época de aquisição das imagens.

\begin{tabular}{|c|c|c|c|c|c|c|c|}
\hline \multicolumn{2}{|r|}{ Estágio } & \multicolumn{2}{|c|}{ Avançado } & \multicolumn{2}{|c|}{ Médio } & \multicolumn{2}{|r|}{ Inicial } \\
\hline Época & Banda & Média & Desvio padrão & Média & Desvio padrão & Média & Desvio padrão \\
\hline \multirow{6}{*}{ Dez/Fev } & 1 (azul) & $0,025^{\mathrm{aA}}$ & 0,024 & $0,023^{\mathrm{bA}}$ & 0,007 & $0,031^{\mathrm{cA}}$ & 0,009 \\
\hline & 2 (verde) & $0,031^{\text {a A }}$ & 0,027 & $0,034^{\mathrm{bA}}$ & 0,007 & $0,050^{c A}$ & 0,012 \\
\hline & 3 (vermelho) & $0,023^{\mathrm{a} A}$ & 0,028 & $0,026^{\mathrm{bA}}$ & 0,007 & $0,042^{c A}$ & 0,013 \\
\hline & 4 (infravermelho próximo) & $0,229^{\mathrm{a}}$ & 0,037 & $0,262^{\mathrm{bA}}$ & 0,031 & $0,300^{\mathrm{cA}}$ & 0,041 \\
\hline & 5 (infravermelho médio) & $0,102^{\text {a A }}$ & 0,030 & $0,119^{\mathrm{b} A}$ & 0,014 & $0,161^{c A}$ & 0,031 \\
\hline & 7 (infravermelho médio) & 0,039a A & 0,027 & $0,047^{\mathrm{b} \mathrm{A}}$ & 0,008 & $0,070^{c A}$ & 0,018 \\
\hline \multirow{6}{*}{ Jun/Ago } & 1 (azul) & $0,020^{\mathrm{aA}}$ & 0,003 & $0,025^{\text {b B }}$ & 0,003 & $0,036^{c B}$ & 0,008 \\
\hline & 2 (verde) & $0,019^{a}$ B & 0,005 & $0,031^{\mathrm{bB}}$ & 0,005 & $0,052^{\mathrm{cA}}$ & 0,015 \\
\hline & 3 (vermelho) & $0,015^{a} \mathrm{~B}$ & 0,004 & $0,025^{\mathrm{bA}}$ & 0,005 & $0,058^{c B}$ & 0,020 \\
\hline & 4 (infravermelho próximo) & $0,155^{a}$ B & 0,050 & $0,271^{\text {b B }}$ & 0,039 & $0,253^{c B}$ & 0,057 \\
\hline & 5 (infravermelho médio) & $0,066^{\mathrm{a}} \mathrm{B}$ & 0,025 & $0,121^{\mathrm{b} \mathrm{A}}$ & 0,018 & $0,198^{\mathrm{CB}}$ & 0,057 \\
\hline & 7 (infravermelho médio) & $0,021^{\mathrm{a} B}$ & 0,011 & $0,045^{\mathrm{b} \text { B }}$ & 0,009 & $0,097^{c B}$ & 0,039 \\
\hline
\end{tabular}

Letras maiúsculas comparam a resposta espectral de cada banda para cada estágio sucessional, entre as épocas analisadas. Letras diferentes representam diferença estatística significante e, por tanto, sazonalidade na resposta espectral da banda. Por exemplo, no estágio avançado as bandas 2, 3, 4, 5 e 7 apresentam diferentes respostas de acordo com a época de aquisição das imagens (teste Mann-Whitney com p-valor $<0,01)$. Letras minúsculas comparam a resposta espectral das classes sucessionais para uma mesma banda e época. Letras diferentes representam diferença estatística significante entre as classes sucessionais (Mann-Whitney com $p$-valor $<0,01$ ).
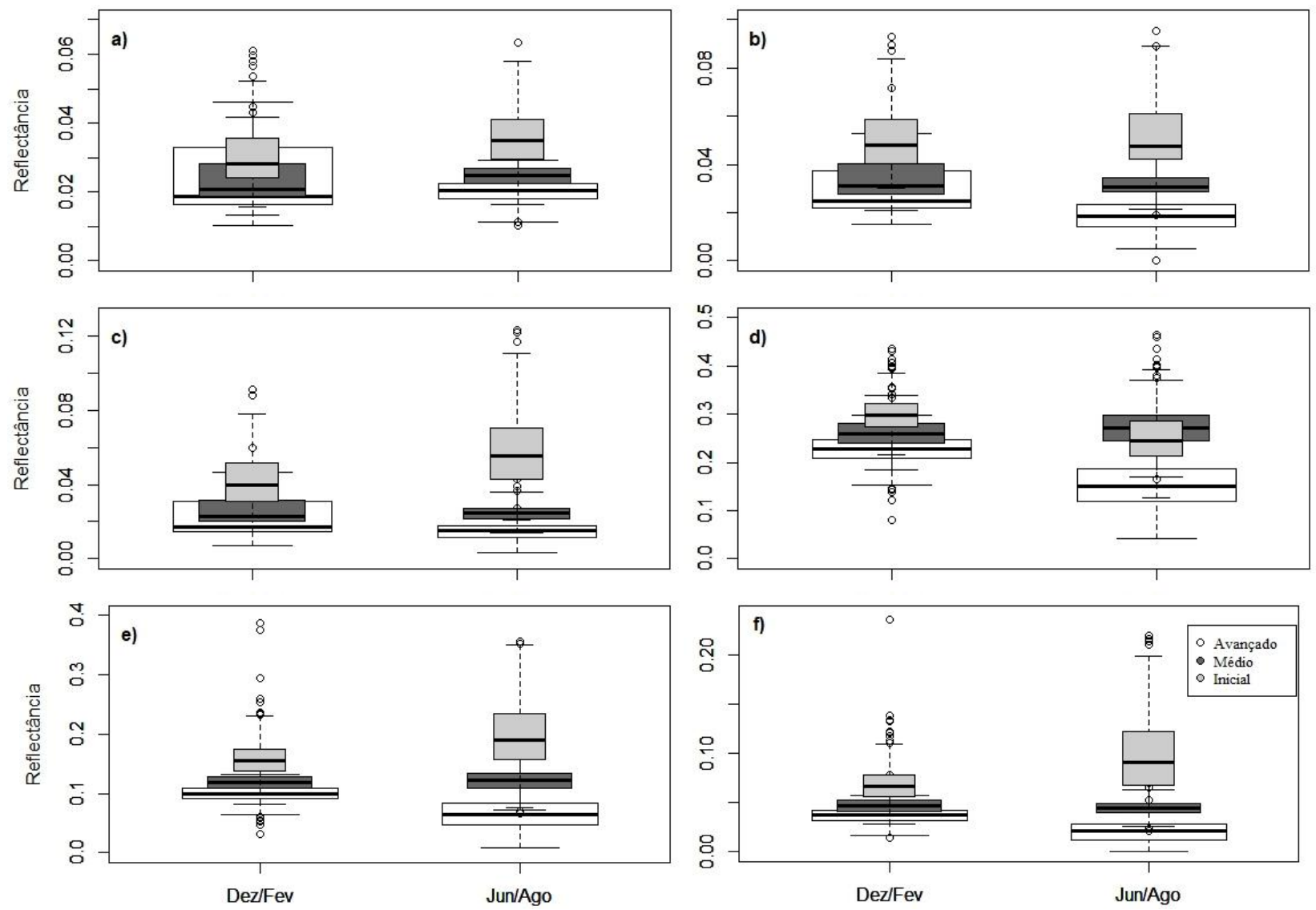

Figura 2. Box-plots da resposta espectral das bandas 1 (a), 2 (b), 3 (c), 4 (d), 5 (e) e 7 (f) de imagens adquiridas de dezembro a fevereiro e de junho a agosto. As caixas englobam $50 \%$ dos dados, do $25^{\circ}$ ao $75^{\circ}$ percentil. A linha central de cada caixa representa a mediana da amostra. As barras englobam $90 \%$ dos dados, do $10^{\circ}$ ao $90^{\circ}$ percentil e estão representadas em largura proporcional a sua respectiva caixa. Outliers estão representados como pequenos círculos. 
Foi previamente demonstrado que o NDVI é afetado por efeitos de saturação quando a cobertura florestal é densa, o que significa que a partir de uma certa densidade florestal, a adição de mais camadas de dossel faz pouca diferença na refletância relativa de radiação vermelha e infravermelha próxima e, portanto, pouca diferença neste IV (GAMON et al., 1995). Isso justifica a ineficiência do NDVI para distinção entre os estágios médio e avançado e confirma que este IV é um mau indicador das características biofísicas em florestas tropicais densas, como já observado em outros estudos (IZQUIERDO et al., 2011; DEVRIES et al., 2015). Desse modo, apesar de sua alta popularidade, o NDVI parece fornecer estimativas consistentes apenas em florestas decíduas e secas.

Assim como o NDVI, o EVI consiste de uma relação na refletância relativa da radiação vermelha e infravermelha próxima, no entanto também inclui fatores de ajuste que o tornam mais robusto para quantificação de biomassa quando comparado ao NDVI (GARROUTE et al., 2016). De acordo com Gurung et al. (2009), o EVI melhora a sensibilidade para as variações estruturais e arquitetônicas do dossel de fitofisionomias com maior densidade de biomassa e minimiza muitos dos problemas de contaminação presentes no NDVI, tais como os associados com o fundo do dossel e com a atmosfera. Os resultados deste estudo concordam com tal afirmativa, uma vez que a distinção entre os estágios médio e avançando obtida pelo EVI foi superior à do NDVI.

O melhor desempenho do NDMI em comparação ao NDVI pode ser explicado pelo seu menor efeito de saturação, o que melhora a distinção de formações florestais de alta densidade (SCHULTZ et al., 2016). Além disso, outros estudos encontraram uma maior sensibilidade da banda 5 (infravermelho médio), utilizada no cálculo do NDMI, para estimar a biomassa de florestas tropicais densas (KENNEDY et al., 2010; ZHU et al., 2012). Segundo Devries et al. (2015), o NDMI é um melhor indicador de maturidade estrutural de floresta nos trópicos, o que pôde ser confirmado neste estudo.

A pós-classificação das amostras de treinamento de acordo com os valores médios dos IV (Tabela 3), confirmou a eficiência observada na análise exploratória dos dados. $O$ NDMI apresentou o melhor percentual de acerto global (68,44\%), seguido pelo NDVI (67,22\%) e EVI (59,33\%). O NDVI e o NDMI obtiveram maior acerto na classificação do estágio inicial (88,67\% e $91 \%$, respectivamente), enquanto o EVI classificou o estágio médio com maior acurácia (91\%).

Os percentuais de acertos obtidos com os três IV variam de moderados a satisfatórios (LANDIS \& $\mathrm{KOCH}, 1977$ ). Dessa forma, a utilização dos mesmos para monitoramento da evolução da vegetação poderá ser eficiente para distinção de alguns estágios sucessionais, ao passo que poderá conduzir a um elevado erro para classificação de outros. De todo modo, as análises realizadas revelam o potencial de distinção entre os estágios sucessionais a partir da resposta espectral das bandas do satélite Landsat 5 TM.

Tabela 2. Média e desvio padrão (SD) dos índices de vegetação para cada classe sucessional.

\begin{tabular}{ccccccc}
\hline Estágio & \multicolumn{2}{c}{ Avançado } & \multicolumn{2}{c}{ Médio } & \multicolumn{2}{c}{ Inicial } \\
\hline Índice & Média & SD & Média & SD & Média & SD \\
\cline { 2 - 7 } NDVI & $0,835^{\mathrm{a}}$ & 0,035 & $0,825^{\mathrm{a}}$ & 0,033 & $0,592^{\mathrm{b}}$ & 0,093 \\
EVI & $0,335^{\mathrm{a}}$ & 0,081 & $0,479^{\mathrm{b}}$ & 0,055 & $0,332^{\mathrm{a}}$ & 0,074 \\
NDMI & $0,385^{\mathrm{a}}$ & 0,050 & $0,356^{\mathrm{b}}$ & 0,051 & $0,063^{\mathrm{c}}$ & 0,109 \\
\hline
\end{tabular}

Letras diferentes representam diferença estatística significante do índice de vegetação entre as classes sucessionais (MannWhitney com p-valor $<0,01$ ).

\section{Análise discriminante quadrática (QDA)}

A classificação das amostras de treinamento a partir da QDA resultou na melhor acurácia entre os métodos de classificação testados, com acerto global de 92,44\%. Em todas as classes sucessionais foi obtido acerto maior que $90 \%$, mostrando a eficiência do classificador quadrático na identificação dos estágios (Tabela 3).

Tabela 3. Percentual de acerto obtido a partir da matriz de validação cruzada da classificação da vegetação feita através dos índices de vegetação NDVI, EVI e MDI e da análise discriminante quadrática (QDA).

\begin{tabular}{ccccc}
\hline Estágio Sucessional & NDVI & EVI & NDMI & QDA \\
\hline Inicial (\%) & 88,67 & 58,67 & 91 & 95 \\
Médio (\%) & 53,33 & 91 & 57,33 & 91 \\
Avançado (\%) & 59,67 & 28,33 & 57 & 91,33 \\
\hline Acerto Global (\%) & $\mathbf{6 7 , 2 2}$ & $\mathbf{5 9 , 3 3}$ & $\mathbf{6 8 , 4 4}$ & $\mathbf{9 2 , 4 4}$ \\
\hline
\end{tabular}

A eficiência da classificação a partir da QDA pode ser explicada pelo fato de a análise ter sido realizada utilizando a informação das seis bandas analisadas no estudo, de forma a maximizar a variância entre as classes consideradas. Thessler et al. (2008), também encontraram resultados promissores com dados do Landsat 5 TM para discriminação de diferentes fisionomias florestais a partir 
de Análise Discriminante.

\section{CONCLUSÕES}

Este estudo mostrou que é possível utilizar a resposta espectral das bandas do satélite Landsat 5 TM para identificação e classificação dos estágios sucessionais em Florestas Ombrófilas Densas.

Apesar de se tratar de um ambiente onde não ocorre déficit hídrico ao longo do ano, existe sazonalidade na resposta espectral da cobertura vegetal, sendo que a mesma deve ser considerada em estudos que utilizem sensoriamento remoto na região. Neste caso, as imagens obtidas no período de menor incidência de chuvas permitem uma melhor distinção entre os estágios sucessionais.

Dentre os Índices de vegetação avaliados (NDVI, EVI e NDMI), o NDMI apresenta melhores resultados para identificação e distinção das classes sucessionais da floresta. No entanto, a Análise discriminante quadrática é ainda mais eficiente que o NDMI para diferenciação do estágio de desenvolvimento da vegetação em Florestas Ombrófilas Densas.

\section{AGRADECIMENTOS}

Agradecemos ao Programa de Pós-Graduação em Engenharia Florestal da Universidade Federal do Paraná (PPGEF / UFPR) pela oportunidade de refinar os estudos da primeira autora e à Coordenação de Aperfeiçoamento de Pessoal de Nível Superior (CAPES) pela concessão de bolsa de doutorado.

\section{REFERÊNCIAS}

AGUIAR, O.T. et al. Flora fanerogâmica de um trecho da floresta densa secundária no Parque Estadual da Serra do Mar - Núcleo Cunha/Indaiá - Cunha (SP). Revista Instituto Florestal, v.13, n.1, p.1-18, 2001.

ALVES, D.S. et al. The changing rates and patterns of deforestation and land use in Brazilian Amazonia. Geophysical Monograph Series, v.186, p.11-23, 2009.

ARCOVA, F.C.S. et al. Ocorrência de nevoeiros em uma microbacia experimental na Serra do Mar, Cunha, SP. Revista Brasileira de Climatologia, v.18, n.12, p.327-348, 2016.

ARMANI, G. Interações entre a atmosfera e a superfície terrestre: variações da temperatura e umidade na bacia $B$ do Núcleo Cunha (IF) - SP. 2004. 198p. (Dissertação de mestrado).

CHANDER, G. et al. Summary of current radiometric calibration coefficients for Landsat MSS, TM, ETM+, and EO-1 ALI sensors.
Remote Sensing of Environment, v.113, n.5, p.893-903, 2009.

CHAVEZ, P.S. An improved dark-object subtraction technique for atmospheric scattering correction of multispectral data. Remote Sensing of Environment, v.24, n.3, p.459-479, 1988.

CICCO, L.S. Evolução da regeneração natural de floresta ombrófila densa alto-montana e a produção de água em microbacia experimental, Cunha -SP. 2013. 61p. (Dissertação de mestrado).

CICCO, V. Determinação da evapotranspiração pelos métodos dos balanços hídrico e de cloreto e a quantificação da interceptação das chuvas na Mata Atlântica: São Paulo, SP e Cunha, SP. 2009. 138p. (Tese de doutorado).

DEVRIES, B. et al. Robust monitoring of small-scale forest disturbances in a tropical montane forest using Landsat time series. Remote Sensing of Environment, v.161, p.107-121, 2015.

FURIAN, S.M.; PFEIFER, R.M. Levantamento de reconhecimento do meio físico do Núcleo Cunha, SP. Boletim Técnico do Instituto Florestal, v.40, n.2, p.183-193, 1986.

FURIAN, S.M. Estudo geomorfológico do escoamento superficial pluvial em parcelas experimentais no Parque Estadual da Serra do Mar - Núcleo Cunha / SP: um esboço metodológico. 1987. 187p. (Dissertação de mestrado).

GAMON, J.A. et al. Relationships between NDVI, canopy structure, and photosynthesis in three Californian vegetation types. Ecological Applications, v.5, n.1, p.28-41, 1995.

GARDNER, T.A. et al. A multi-region assessment of tropical forest biodiversity in a human-modified world. Biological Conservation, v.143, n.10, p.2293-2300, 2010.

GARROUTE, E.L. et al. Using NDVI and EVI to map spatiotemporal variation in the biomass and quality of forage for Migratory Elk in the Greater Yellowstone ecosystem. Remote Sensing, v.8, n.5, p.1-25, 2016.

GURUNG, R.B. et al. Predicting enhanced vegetation index (EVI) curves for ecosystem modeling applications. Remote Sensing of Environment, v.113, n.10, p.2186-2193, 2009.

IBGE - INSTITUTO BRASILEIRO DE GEOGRAFIA E ESTATÍSTICA. Manual técnico da vegetação brasileira. 2. ed. Rio de Janeiro: Departamento de Cartográfica, 2012.

IMANI, M.; GHASSEMIAN, H. Feature space discriminant analysis for hyperspectral data feature reduction. ISPRS Journal of Photogrammetry and Remote Sensing, v.102, p.1-13, 2015.

IZQUIERDO, T. et al. Vegetation indices changes in the cloud forest of La Gomera Island (Canary Islands) and their hydrological implications. Hydrological Process, v.25, n.10, p.1531-1541, 2011.

KENNEDY, R.E. et al. Detecting trends in forest disturbance and recovery using yearly Landsat time series: 1. LandTrendr Temporal segmentation algorithms. Remote Sensing of Environment, v.114, p.2897-2910, 2010. 
LANDIS, J.R.; KOCH, G.G. The measurement of observer agreement for categorical data. Biometrics, v.33, n.1, p.159-174, 1977.

LIN, C. et al. A novel reflectance-based model for evaluating chlorophyll concentrations of fresh and water-stressed leaves. Biogeosciences, v.12, p.49-66, 2015.

MANLY, B.F.J. Métodos estatísticos multivariados: uma introdução. 3.ed. Porto Alegre: Bookman, 2008.

MARKHAM, B.L.; BARKER, J.L. Thematic mapper bandpass solar exoatmospheric irradiances. International Journal of Remote Sensing, v.8, n.3, p.517-523, 1987.

NEPSTAD, D.C. et al. The role of deep roots in the hydrological and carbon cycles of Amazonian forests and pastures. Nature, v.372, p.666-669, 1994.

RANZINI, M. et al. Geração do deflúvio de uma microbacia com Mata Atlântica, Cunha, SP. Revista Instituto Florestal, v.23, n.2, p.179-190, 2011.

RIBEIRO, M.C. et al. The Brazilian Atlantic Forest: how much is left, and how is the remaining forest distributed? Implications for conservation. Biological Conservation, v.142, n.6, p.1141-1153, 2009.

SCHULTZ, M. et al. Performance of vegetation indices from Landsat time series in deforestation monitoring. International Journal of Applied Earth Observation and Geoinformation, v.52, p.318-327, 2016.

THESSLER, S. et al. Using k-nn and discriminant analyses to classify rain forest types in a Landsat TM image over northern Costa Rica. Remote Sensing of Environment, v.112, n.5, p.2485-2494, 2008.

ZHANG, C. et al. Photochemical reflectance index (PRI) for detecting responses of diurnal and seasonal photosynthetic activity to experimental drought and warming in a Mediterranean Shrubland. Remote Sensing, v.9, n.11, p.1189-1210, 2017.

ZHU, Z. et al. Continuous monitoring of forest disturbance using all available Landsat imagery. Remote Sensing of Environment, v.122, p.75-91, 2012. 